\title{
Chairman's closing remarks
}

By N. Veall, Radioisotopes Division, Clinical Research Centre, Harrow, Middlesex

Dr Nolan mentioned the use of ${ }^{51} \mathrm{Cr}$ EDTA as an inert rumen marker. In clinical work the use of $\mathrm{Cr}_{2} \mathrm{O}_{3}$ with all its disadvantages is still widespread. I can confirm from my own experience that ${ }^{51} \mathrm{Cr}$ EDTA is very useful for gastrointestinal studies (as well as for the measurement of glomerular filtration rate) in man. I personally, and probably many other clinical workers, feel obliged to the Australian veterinarians, as represented by Downes \& McDonald (1964), for drawing attention to the useful properties of the material. Dr Nolan also drew attention to the value of computer modelling for data fitting in tracer studies in nutrition. Perhaps I should emphasize that analogue computer simulation of biological systems of the kind discussed today is assuming major importance as the best available method for the interpretation of results obtained under non-steady-state conditions. In general, the mathematical analyses described today have depended on the existence of steady state conditions, which are often difficult to achieve in practice. More important, studies under non-steady-state conditions are highly desirable if one is to gain insight into the control systems responsible for proper function or homoeostasis in a particular biological system.

The paper by Dr Horsfield and his colleagues is representative of many studies of its type, which depend ultimately on multi-exponential curve analysis. There is no doubt that very close curve fitting can be achieved with computer programmes such as the SAAM 25. However, in real life situations one is fitting data points with a finite experimental error. This can result in very large standard errors on the numerical values for the various curve parameters. In practice, this technique can be useful for up to three components, provided the data are sufficiently accurate and the values of the exponents differ considerably. Anyone embarking on this sort of exercise is advised to read the paper by Glass \& de Garreta (197I) on the quantitative limitations of exponential curve fitting.

\section{REFERENCES}

Downes, A. M. \& McDonald, I. W. (1964). Br. J. Nutr. 18, 53. Glass, H. I. \& de Garreta, A. C. (1971). Phys. Med. Biol. 16, x 19. 